\title{
Human papillomavirus type 16 E6 variants in France and risk of viral persistence
}

\author{
Iris Cornet ${ }^{1 *}$, Tarik Gheit ${ }^{1}$, Gary M Clifford ${ }^{1}$, Jean-Damien Combes $^{1}$, Véronique Dalstein ${ }^{2}$, Silvia Franceschi ${ }^{1}$, \\ Massimo Tommasino ${ }^{1}$ and Christine Clavel $^{2}$
}

\begin{abstract}
Background: Only a small portion of HPV 16 infections persist and can lead to cervical intraepithelial lesions and cancer. Factors that favour HPV persistence versus clearance are still poorly understood, but several studies have suggested that HPV intra-type variants may influence persistence and clinical outcome. The aim of this study was to assess the possible association between HPV 16 variants and the risk for viral persistence in the general population of France.

Methods: One hundred and forty two women infected with HPV 16 with normal cytology, without previous treatment for cervical lesions, and with a valid second follow-up visit 4 to 16 months later, were selected from patients participating in routine cervical cancer screening in the Reims HPV Primary Screening Cohort Study. HPV intra-type variants were determined by sequencing the HPV 16 E6 open reading frame, and were compared for viral persistence at the second visit using odds ratios (OR) to estimate relative risk.
\end{abstract}

Results: Although no statistically significant differences in risk for persistence were observed by the HPV 16 variant lineage, European variants containing the polymorphism $350 \mathrm{~T}$ (EUR-350 T) appeared to persist more often than those containing $350 \mathrm{G}$ (EUR-350 G) $(\mathrm{OR}=1.6,95 \% \mathrm{Cl}=0.8-3.4)$.

Conclusions: No strong differences were observed in the risk of viral persistence for the HPV 16 variants that predominate in France.

Keywords: Cervical cancer screening, Cohort, Polymorphism, HPV, Variants, Persistence

\section{Background}

High-risk (HR) human papillomaviruses (HPVs), are the etiological agents of cervical cancer [1]. Even though infection with an HR-HPV is frequent in sexually active women, the majority of infections are resolved by the immune system in 6-12 months [2]. Only a small fraction of infections persist and can lead to cervical intraepithelial neoplasia (CIN), which may progress to cervical cancer. Factors that favour HPV persistence versus clearance, other than HPV type itself, are still poorly understood, but several studies have suggested that HPV intra-type variants could influence persistence and clinical outcome [3-8].

\footnotetext{
* Correspondence: icb@iarc.fr

${ }^{1}$ International Agency for Research on Cancer, 150 cours Albert Thomas, Lyon 69372 Cedex 08, France

Full list of author information is available at the end of the article
}

A recent proposal to define major HPV variant lineages is by approximately $1.0 \%$ difference between full genomes of the same HPV type, with differences of $0.5-0.9 \%$ designated as sub-lineages [9-11]. Naturally occurring genetic variants of HPV 16 are common and have previously been classified into 4 major lineages; European-Asian, including the sub-lineages European (EUR), and Asian (As), African 1 (AFR1), African 2 (AFR2) and North-American/AsianAmerican (NA/AA). The names of the (sub)lineages derive from the geographical origin of the populations in which they were originally isolated [12].

The most common individual polymorphism within the European lineage is T350G in the E6 oncogene, which leads to an amino acid change Leucine to Valine (L83V). Thus, the European lineage can be divided into isolates containing $350 \mathrm{~T}$ (EUR-350 T) or $350 \mathrm{G}$ (EUR$350 \mathrm{G}$ ), even though this polymorphic position does not seem to define phylogenetic lineages [10]. Several studies 
suggest that the EUR-350 T and EUR-350 G have a different distribution among cervical cancer cases and controls and can influence persistence $[3,13,14]$.

The aim of this study was to assess the possible association between the genetic variants of HPV 16 and the risk of persistent HPV infection in the general population in France.

\section{Results}

Sequencing results were obtained for a total of 142 women infected with HPV 16 with normal cytology and a valid follow-up visit. The follow-up visits ranged from minimum 4.0 months to maximum 16.0 months (median: 7.7 months, 25 th percentile: 6.2 months, 75 th percentile: 10.9 months). The majority of the baseline infections belonged to the European lineage $(n=131,92.3 \%)$, with EUR-350 $\mathrm{T}$ and EUR-350 G accounting for $40.8 \%$ and $51.4 \%$ of the isolates, respectively. C109T $(n=3)$ was the only other single nucleotide polymorphism (SNP) found in more than one EUR isolate (Table 1). The number of non-EUR isolates was small $(n=11)$ and consisted of African-lineages and NA-lineage isolates (Table 1).

Of these 142 women infected with HPV 16, 86 (60.6\%) showed a persistent infection at the second visit. The proportion of HPV 16 infections that persisted was slightly higher for EUR-350 $\mathrm{T}(67.2 \%)$ than EUR-350 G (56.1\%) $(\mathrm{OR}=1.6,95 \% \mathrm{CI}=0.7-3.5)$, although this difference did not reach statistical significance. Adjustment for age and time to second visit did not materially alter this finding $(\mathrm{OR}=1.6,95 \% \mathrm{CI}=0.8-3.4) \quad$ (Table 2). Among the $32 \mathrm{HPV} 16$ infections that had evidence of persistence for 12 months and more, 16 (50.0\%) were EUR-350 T and 13 (40.6\%) EUR-350 G (OR versus clearance $=2.1,95 \% \mathrm{CI}=0.7-5.8$, data not shown).

Due to the rarity of non-European lineages a comparison by individual lineage could not be made. However, the proportion of all non-European lineages combined that persisted was similar to that for EUR-350 G (OR = 0.9, $95 \% \mathrm{CI}=0.2-3.4)($ Table 2$)$.

\section{Discussion}

There were no statistically significant differences in the risk for persistence by HPV 16 variant lineage among women participating to the Reims HPV Primary Screening Cohort Study between 2001 and 2008. This finding, together with the distribution of the HPV 16 variants among infections at baseline, is consistent with results of a smaller and earlier study from this same cohort [14]. Nevertheless, European lineage isolates containing the polymorphism $350 \mathrm{~T}$ (EUR-350 T) did appear to persist more often than those containing $350 \mathrm{G}$ (EUR-350 G) $(\mathrm{OR}=1.6,95 \% \mathrm{CI}=0.8-3.4)$. This is consistent with the findings of the only other population-based study of HPV 16 variant persistence in Europe: among $261 \mathrm{HPV}$
16 infections in a large cohort in Denmark, infection with EUR-350 $\mathrm{T}$ was associated with a significant increased risk for persistence at 2 years (OR versus EUR-350 G $=2.06,95 \% \mathrm{CI}=1.04-4.25$ ) [3].

One weakness of the present study was the inability to make any robust reflection on viral persistence of nonEUR HPV 16 lineages, due to their rarity in the French population $[4,7,15]$. Furthermore, the time-interval to determine infection persistence was relatively short, driven by the use of samples from a real-life screening practice in which women with an HR-HPV infection with normal cytology were advised to return at 6-12 months.

As a consequence of viral persistence, it would be expected to find the EUR-350 T more often in cervical cancer. In accordance, Zehbe et al. showed an enrichment of EUR-350 $\mathrm{T}$ in invasive cervical cancer in the Czech Republic, and, to a lesser extent, in Italy. However, they also found a significant under-representation of EUR-350 T in invasive cervical cancer in Sweden [13]. This suggests that the relative oncogenic potential of HPV 16 EUR-350 $\mathrm{T}$ versus EUR-350 G might be population-specific [13].

\section{Conclusions}

Both EUR-350 T and EUR-350 G are found commonly in precursor lesions and cervical cancers in Northern Europe, meaning that any clinical utility of variant analysis is not yet evident. Nevertheless, understanding the genetic basis of differences in persistence of HPV 16 variants may help to unravel important interactions between HPV 16 and the host immune system. This could lead to better tools to control HPV infections and its associated malignancies.

\section{Methods}

\section{Study population}

Samples were selected from patients participating in the Reims HPV Primary Screening Cohort Study conducted in clinics and hospitals in Reims, France, and its surroundings $[16,17]$. Briefly, since 1997, women participating in the study were offered systematic cervical cancer screening combining liquid based cytology and HR-HPV testing by the Hybrid Capture 2 Test (Qiagen, Gaithersburg, MD USA) when they had their routine cervical smear performed. Women positive for HR-HPV or with abnormal cytology were advised to return 6-12 months later. For research purposes, since 2001, DNA has been extracted routinely from HR-HPV positive samples and screened for HPV 16 presence. The Reims HPV Primary Screening Cohort Study has been approved by the French committee "Comité de Protection des Personnes" (CPP-Est III, Nancy), statement no DC-2008-374. All women were informed and gave their written consent to participate in the study. 
Table 1 Nucleotide sequence variations in the HPV 16 E6 gene of 142 HPV 16-positive French women

\begin{tabular}{|c|c|c|c|c|c|c|c|c|c|c|c|c|c|c|c|c|c|c|c|c|c|c|c|c|c|c|}
\hline \multirow{2}{*}{$\frac{\text { Status }}{\text { Clearance* }^{*}}$} & \multirow[b]{2}{*}{ Persistence* } & \multicolumn{24}{|c|}{ HPV 16 E6 nucleotide position } & \multirow[b]{2}{*}{ (sub-) lineage } \\
\hline & & 109 & 131 & 132 & 143 & 145 & 176 & 183 & 187 & 196 & 255 & 256 & 269 & 275 & 285 & 286 & 289 & 295 & 335 & 350 & 403 & 453 & 464 & 478 & 532 & \\
\hline $\mathrm{N}$ & $\mathbf{N}$ & $\bar{T}$ & $\bar{A}$ & $\bar{G}$ & $\mathrm{C}$ & $\bar{G}$ & $\bar{G}$ & $T$ & A & $\bar{G}$ & G & $\mathrm{C}$ & $\bar{G}$ & $\bar{A}$ & C & $T$ & A & $\bar{T}$ & C & $\bar{T}$ & A & G & A & $\bar{C}$ & A & Reference \\
\hline 13 & 29 & - & - & - & - & - & - & - & - & - & - & - & - & - & - & - & - & - & - & - & - & - & - & - & - & EUR-350 T \\
\hline 6 & 6 & 0 & 0 & 0 & 0 & 0 & 0 & 0 & 0 & 0 & 0 & - & - & - & - & - & - & - & - & - & 0 & 0 & 0 & 0 & 0 & EUR-350 T \\
\hline 0 & 1 & - & - & - & - & - & C & - & - & - & - & - & - & - & - & - & - & - & - & - & - & - & - & - & - & EUR-350 T \\
\hline 0 & 1 & - & - & - & - & - & - & - & G & - & - & - & - & - & - & - & - & - & - & - & - & - & - & - & - & EUR-350 T \\
\hline 0 & 1 & - & - & - & - & - & - & - & - & - & - & - & A & - & - & - & - & - & - & - & - & - & - & - & - & EUR-350 T \\
\hline 0 & 1 & - & - & - & - & - & - & - & - & - & - & - & - & - & - & - & - & - & - & - & - & - & G & - & - & EUR-350 T \\
\hline 22 & 27 & - & - & - & - & - & - & - & - & - & - & - & - & - & - & - & - & - & - & G & - & - & - & - & - & EUR-350 G \\
\hline 9 & 9 & 0 & 0 & 0 & 0 & 0 & 0 & 0 & 0 & 0 & 0 & - & - & - & - & - & - & - & - & G & 0 & 0 & 0 & 0 & 0 & EUR-350 G \\
\hline 0 & 3 & C & - & - & - & - & - & - & - & - & - & - & - & - & - & - & - & - & - & G & - & - & - & - & - & EUR-350 G \\
\hline 0 & 1 & - & - & - & - & - & - & - & - & - & - & - & - & - & - & - & - & - & - & G & - & $\mathrm{T}$ & - & - & - & EUR-350 G \\
\hline 0 & 1 & - & - & - & - & - & - & - & - & - & - & - & - & - & - & - & - & - & - & G & - & - & - & A & - & EUR-350 G \\
\hline 1 & 0 & - & - & - & - & - & - & - & - & - & - & - & - & G & - & - & - & - & - & G & - & - & - & - & - & EUR-350 G \\
\hline 0 & 1 & - & - & - & - & $\mathrm{T}$ & - & - & - & - & - & - & - & - & - & A & G & - & $\mathrm{T}$ & G & - & - & - & - & - & NA (non-EUR) \\
\hline 1 & 1 & - & - & C & G & $\mathrm{T}$ & - & - & - & - & - & - & - & - & - & A & G & - & $\mathrm{T}$ & - & - & - & - & - & - & AFR1 (non-EUR) \\
\hline 0 & 1 & - & G & - & G & T & - & - & - & - & - & - & - & - & - & A & G & G & T & G & - & - & - & - & - & AFR1 (non-EUR) \\
\hline 2 & 1 & C & - & T & G & T & - & - & - & - & - & - & - & - & - & A & G & - & $\mathrm{T}$ & - & G & - & - & - & - & AFR2 (non-EUR) \\
\hline 1 & 0 & C & - & T & G & T & - & C & - & - & - & - & - & - & - & A & G & - & T & - & G & - & - & - & - & AFR2 (non-EUR) \\
\hline 0 & 1 & C & - & T & G & T & - & - & - & - & - & - & - & - & G & A & G & - & T & - & G & - & - & - & - & AFR2 (non-EUR) \\
\hline 0 & 1 & - & - & - & G & T & - & - & - & A & $\mathrm{T}$ & $\mathrm{T}$ & - & - & - & A & G & G & $\mathrm{T}$ & - & - & - & - & - & - & AFR2 (non-EUR) \\
\hline 1 & 0 & - & - & - & G & T & - & - & - & - & - & - & - & - & - & A & G & - & T & - & - & - & - & - & - & AFR2 (non-EUR) \\
\hline 56 & 86 & & & & & & & & & & & & & & & & & & & & & & & & & \\
\hline
\end{tabular}

Sequence results are shown by outcome of infection: *Clearance (HPV16-negative at follow-up visit between 4-16 months) or *Persistence (HPV16-positive at follow-up visit between 4-16 months). Abbreviations: EUR EURopean, NA North American, AFR AFRican.

$0=$ failed sequencing for the whole E6 gene, a shorter fragment of E6 was determined (nt. 256-400). 
Table 2 Outcome of HPV 16 infection in 142 French women, by HPV 16 variant lineage

\begin{tabular}{lcccc}
\hline Variant & \multicolumn{2}{c}{ Clearance* } & & \multicolumn{2}{c}{ Persistence* } \\
\cline { 2 - 3 } \cline { 5 - 5 } & $\mathbf{n}(\%)$ & & $\mathbf{n}(\%)$ & $\mathbf{a O R}^{\dagger}[\mathbf{9 5 \%} \mathbf{C l}]$ \\
\hline EUR-350 G & $32(43.8)$ & & $41(56.1)$ & 1 \\
EUR-350 T & $19(32.8)$ & & $39(67.2)$ & $1.6[0.8-3.4]$ \\
Non-EUR & $5(45.5)$ & & $6(54.5)$ & $0.9[0.2-3.4]$ \\
\hline
\end{tabular}

* Clearance = HPV 16-negative at follow-up visit; Persistence = HPV 16-positive at follow-up visit.

${ }^{\dagger}$ aOR: Odds Ratio adjusted for age and time interval between first and followup visit.

For the period 2001 to 2008, HPV 16-positive women that met the following criteria were selected: diagnosed with normal cytology and with a valid stored DNA sample at their first HPV 16-positive visit, without previous treatment for cervical lesions, and with a valid follow-up visit of known HPV 16 DNA between 4 to 16 months later.

These patients overlap partially $(n=21)$ with those included in a previous report on HPV 16 variants from this same cohort between 1997 and 2003 [14].

\section{HPV testing and sequencing}

HPV 16 E6 variants were analysed from DNA stored from the first HPV 16-positive normal cytology sample. DNA was extracted from liquid-based cervical samples, collected in PreservCyt Medium (Hologic, Marlborough, MA USA), with the EZ1 DNA Tissue Kit on the EZ1 instrument (Qiagen, Hilden, Germany), according to the manufacturer's instructions. Samples were lysed overnight using proteinase $\mathrm{K}$ at $56^{\circ} \mathrm{C}$ and DNA was eluted in a $200 \mu \mathrm{L}$ volume.

HPV 16 DNA presence was screened initially by a specific HPV 16 E6/E7 Taqman real-time polymerase chain reaction (PCR) and confirmed by either the Linear Array HPV Genotyping Test (Roche Molecular Diagnostics, Branchburg, NJ USA) or the Inno-Lipa Extra HPV Genotyping Test (Innogenetics, Gent, Belgium).

The PCR and sequencing of the HPV 16 E6 gene was performed as described by Gheit et al. 2011 [3]. If amplification of the whole HPV 16 E6 failed, a shorter fragment of E6 (nt. 256 - 400) was amplified with the primers: 5'- GACTTTGCTTTTCGGGATT -3' and 5'GGCTTTTGACAGTTAATAC -3'. The sequences were analyzed using the Blast function from PubMed and single nucleotide polymorphisms (SNP) were identified using the prototype HPV 16 sequence described by Myers et al. [18]. Novel SNPs were confirmed by a second, independent PCR and sequencing reaction. Data were validated by comparing the SNPs reported by visual inspection in Blast with the output files from the DNA sequencer, using an ad hoc designed computer program.

\section{Statistical analysis}

The HPV 16 infection was defined as persistent if the woman was still HPV 16-positive at the next follow-up visit. Relative risks for persistence were compared between EUR-350 T, EUR-350 G, and non-European lineages, using odds ratios (OR) and corresponding 95\% confidence intervals (CI). ORs were adjusted for age $(<28$ and $28+$ years) and the duration between two visits ( $<8$ and $8+$ months).

\section{Abbreviations}

HR-HPV: High Risk Human Papillomavirus; CIN: Cervical Intra-epithelial Neoplasia; EUR: European; As: Asian; AFR: African; NA/AA: North-American / Asian-American; PCR: Polymerase Chain Reaction; SNP: Single Nucleotide Polymorphism; OR: Odds Ratio; Cl: Confidence Interval.

\section{Competing interests}

The authors declare that they have no competing interests.

\section{Authors' contributions}

VD and CC are responsible for the Reims screening study and research databases and participated in the study design. IC performed the HPV variants testing and lead drafting of the manuscript. TG developed the HPV variant testing platform and performed variant testing. MT developed the HPV variant testing platform and participated in the data interpretation. JC participated in the data analysis and performed statistical analysis. GC participated in the study design and interpretation. SF participated in study interpretation. All authors read and approved the final manuscript.

\section{Acknowledgements}

This work was supported by grants from The Association for International Cancer Research (AICR), UK (project grant number 08-0213), Institut National du Cancer (INCa), France (collaboration agreement 07/3D1514/PL-89-05/NGLC), Fondation Innovations en Infectiologie (FINOVI; project no. AO1-project 2) and the European Commission, grant HPV-AHEAD (FP7-HEALTH-2011282562). The authors thank Vanessa Tenet, Jerome Vignat, Annick Rivoire, and Veronique Chabanis. for their technical and administrative support.

\section{Author details}

'International Agency for Research on Cancer, 150 cours Albert Thomas, Lyon 69372 Cedex 08, France. ${ }^{2}$ INSERM UMR-S 903 / Université de Reims Champagne-Ardenne / CHU Reims, Laboratoire Pol-Bouin, Reims, France.

Received: 18 October 2012 Accepted: 17 December 2012 Published: 23 January 2013

\section{References}

1. zur Hausen H: Papillomaviruses and cancer: from basic studies to clinical application. Nat Rev Cancer 2002, 2(5):342-350.

2. Maucort-Boulch D, Plummer M, Castle PE, Demuth F, Safaeian M, Wheeler CM, Schiffman M: Predictors of human papillomavirus persistence among women with equivocal or mildly abnormal cytology. Int J Cancer 2010, 126(3):684-691.

3. Gheit T, Cornet I, Clifford GM, Iftner T, Munk C, Tommasino M, Kjaer SK: Risks for persistence and progression by human papillomavirus type 16 variant lineages among a population-based sample of Danish women. Cancer Epidemiol Biomarkers Prev 2011, 20(7):1315-1321.

4. Schiffman M, Rodriguez AC, Chen Z, et al: A population-based prospective study of carcinogenic human papillomavirus variant lineages, viral persistence, and cervical neoplasia. Cancer Res 2010, 70(8):3159-3169.

5. Zuna RE, Moore WE, Shanesmith RP, Dunn ST, Wang SS, Schiffman M, Blakey GL, Teel T: Association of HPV16 E6 variants with diagnostic severity in cervical cytology samples of 354 women in a US population. Int J Cancer 2009, 25(11):2609-2613.

6. Sathish N, Abraham P, Peedicayil A, Sridharan G, Chandy G: HPV 16 E6 sequence variations in Indian patients with cervical neoplasia. Cancer Lett 2005, 229(1):93-99.

7. Villa LL, Sichero L, Rahal P, Caballero O, Ferenczy A, Rohan T, Franco EL: Molecular variants of human papillomavirus types 16 and 18 
preferentially associated with cervical neoplasia. J Gen Virol 2000, 81(Pt 12):2959-2968.

8. Berumen J, Ordonez RM, Lazcano E, et al: Asian-American variants of human papillomavirus 16 and risk for cervical cancer: a case-control study. J Natl Cancer Inst 2001, 93(17):1325-1330.

9. Burk RD, Chen Z, Harari A, Smith BC, Kocjan BJ, Maver PJ, Poljak M: Classification and nomenclature system for human alphapapillomavirus variants: general features, nucleotide landmarks and assignment of hpv6 and hpv 11 isolates to variant lineages. Acta Dermatovenerol Alp Panonica Adriat 2011, 20(3):113-123.

10. Chen Z, Terai M, Fu L, Herrero R, Desalle R, Burk RD: Diversifying selection in human papillomavirus type 16 lineages based on complete genome analyses. J Virol 2005, 79(11):7014-7023.

11. Chen Z, Schiffman M, Herrero R, et al: Evolution and taxonomic classification of Human Papillomavirus 16 (HPV16)-related variant genomes: HPV31, HPV33, HPV35, HPV52, HPV58 and HPV67. PLoS One 2011, 6(5):e20183.

12. Yamada T, Manos MM, Peto J, Greer CE, Munoz N, Bosch FX, Wheeler CM: Human papillomavirus type 16 sequence variation in cervical cancers: a worldwide perspective. J Virol 1997, 71(3):2463-2472.

13. Zehbe I, Tachezy R, Mytilineos J, et al: Human papillomavirus 16 e6 polymorphisms in cervical lesions from different European populations and their correlation with human leukocyte antigen class ii haplotypes. Int J Cancer 2001, 94(5):711-716.

14. Grodzki M, Besson G, Clavel C, Arslan A, Franceschi S, Birembaut P, Tommasino M, Zehbe I: Increased risk for cervical disease progression of French women infected with the human papillomavirus type 16 e6$350 \mathrm{~g}$ variant. Cancer Epidemiol Biomarkers Prev 2006, 15(4):820-822.

15. Xi LF, Koutsky LA, Hildesheim A, Galloway DA, Wheeler CM, Winer RL, Ho J, Kiviat NB: Risk for high-grade cervical intraepithelial neoplasia associated with variants of human papillomavirus types 16 and 18. Cancer Epidemiol Biomarkers Prev 2007, 16(1):4-10.

16. Clavel C, Masure M, Bory JP, et al: Human papillomavirus testing in primary screening for the detection of high-grade cervical lesions: a study of 7932 women. Br J Cancer 2001, 84(12):1616-1623.

17. Clavel C, Cucherousset J, Lorenzato $M$, et al: Negative human papillomavirus testing in normal smears selects a population at low risk for developing high-grade cervical lesions. Br J Cancer 2004, 90(9):1803-1808.

18. Myers G, Delius H, Icenogle J, Bernard HU, Baker C, Halpern A, Wheeler C: Human Papillomaviruses 1995: A compilation and analysis of nucleic acid and amino acid sequences. Los Alamos, NM: Los Alamos National Library; 1995.

doi:10.1186/1750-9378-8-4

Cite this article as: Cornet et al:: Human papillomavirus type 16 E6 variants in France and risk of viral persistence. Infectious Agents and Cancer 2013 8:4.

\section{Submit your next manuscript to BioMed Central and take full advantage of:}

- Convenient online submission

- Thorough peer review

- No space constraints or color figure charges

- Immediate publication on acceptance

- Inclusion in PubMed, CAS, Scopus and Google Scholar

- Research which is freely available for redistribution

Submit your manuscript at www.biomedcentral.com/submit
( Biomed Central 